\title{
An Artificial Bee Optimization Based on Command Filtered CDM-Backstepping for Electro-Pneumatic System
}

\author{
Fouad Haouari ${ }^{1 *}$, Rabah Gouri', Nourdine Bali², Mohamed Tadjine³, Mohamed Seghir Boucherit ${ }^{3}$ \\ 1 Department of Industrial Engineering and Maintenance, Ecole Nationale Supérieure de Technologie (ENST), \\ Cité Diplomatique, Ex Centre Biomédical, Dergana, Bordj El Kiffan, 16078 Algiers, Algeria \\ 2 Department of Electrical Engineering, Faculty of Electronics and Computer Science, University of Sciences and Technology \\ Houari Boumediene (USTHB), 16111 Algiers, Bab Ezzouar, P. O. B. 32, Algeria \\ ${ }^{3}$ Process Control Laboratory, Department of Electrical Engineering, Ecole Nationale Polytechnique (ENP), \\ 10 Avenue Hassan Badi, El Harrach, 16200 Algiers, Algeria \\ *Corresponding author, e-mail: haouari_fouad@yahoo.fr
}

Received: 25 March 2019, Accepted: 03 June 2019, Published online: 15 August 2019

\begin{abstract}
The proposed manuscript presents a coefficient diagram method (CDM) controller for an electro-pneumatic system. In order to tune the controller parameters, an artificial bee colony (ABC) optimization method is applied. According to the simulation results, the optimized parameters can provide better dynamic and steady state performances and higher robustness to the control algorithm, than the conventional tuned parameters.
\end{abstract}

\section{Keywords}

artificial bee optimization, command filtered backstepping, coefficients diagram method, pneumatic actuator

\section{Introduction}

Electro-pneumatic systems have many advantages such as low weight and size; these systems are robust, economical with easy installation and maintenance [1-4], they are now used in several industrial sectors such as transporting system, buildings, machine tools, assembly, pharmaceutical and chemical system. Contrariwise, the design of control laws for such systems is not easy due to its difficult modeling such as presence of parametric uncertainty.

Newly, several control approach were proposed to electro-pneumatic systems such as the classical technique proportional-integral-derivative (PID) linear controller, it is widely employed, however this kind is deliberated as a fixed parameters controller which planned at nominal operating points to obtain a linearized model transfer function, this approach is not appropriate in all operating point, because of the incompetence of the PID controller's to the effect of the nonlinearities. The sliding mode control displays its usefulness due to its robustness and the guaranteed convergence. However, its weakness is the well-known "chattering" phenomenon $[2,5]$, produced by the discontinuous input. Backstepping is one of these advanced control techniques that has attracted researcher in recent years.
The main idea of this method is to select some suitable functions of state variables as virtual control variables for subsystems in the structure of the overall system recursively [6-10]. It can evade cancellations of useful nonlinear terms and frequently introduce additional nonlinearities to enhance transient performance, but in this approach, the control law is designed at each step in the procedure by means of virtual control signals and their derivatives which are tedious for controlling nonlinear system when the order system is greater than three because the control signal will include the successive derivative of the virtual control.

The fundamental contribution of this paper is the development of a new command filtered backstepping [11-14] combined with CDM controller [15-18] which will be applied to an electro-pneumatic system for position tracking; this approach requires only the reference signals and theirs derivatives to be available as inputs to the control system. The proposed control can guarantee an asymptotic convergence and enhance the robustness of the control law in the presence of disturbance and parameter variations.

The controller parameters tuning is frequently the main problem in the control field, when the classical command 
filtered CDM-backstepping control is characterized by strong robustness and high stability. In order to achieve the high performance for position tracking, continuous adjustments of controller parameters have to been done by searching the optimal adjustment coefficient within short period. Conventional controller parameters adjusting used the trial and error method, which is complex and the period of tuning is length. In order to enhance this controller and guarantee the optimum performance, a swarm intelligence optimization algorithm namely ABC algorithm [19-21] is used to optimize the parameters of the proposed controller. The main novelty of the proposed approach consists in solving simultaneously both the problem of increasing terms in the traditional CDM-backstepping method caused by the repeated differentiations of virtual control laws and the complexity of tuning many parameters of controller to obtain high performance, it is proven in simulation that the better performance in motion positioning is achieved by using this optimization approach.

The remainder of this paper is structured as follows. Section 2, explains the electro-pneumatic system dynamics in state space model. Section 3 is dedicated to the design procedure of linear control for CDM. Section 4 is devoted to establish the command filtered backstepping based on coefficient diagram method with stability analysis, Section 5 describes the ABC optimization and its application. Section 6 evaluates the performance of the proposed approach using computer simulations. The last section concludes this paper.

\section{Electro-pneumatic system modeling}

The state space model of this system is given in [1-4] by Eq. (1):

$$
\left\{\begin{array}{rl}
\frac{d y}{d t} & =v \\
\frac{d v}{d t} & =\frac{1}{M}\left(S P_{P}-S P_{N}-b_{v} v-F_{e x t}(t)\right) \\
\frac{d P_{P}}{d t} & =\frac{k r T}{V_{P}(y)}\left(q_{m P}\left(u_{P}, P_{P}\right)-\frac{S}{r T} P_{P} v\right) \\
\frac{d P_{N}}{d t} & =\frac{k r T}{V_{N}(y)}\left(q_{m N}\left(u_{N}, P_{N}\right)+\frac{S}{r T} P_{N} v\right)
\end{array} .\right.
$$

Sothefunctions $q_{m P}\left(u_{P}, P_{P}\right)=\varphi\left(P_{P}\right)+\psi\left(P_{P}, \operatorname{sgn}\left(u_{P}\right)\right) u_{P}$, $q_{m N}\left(u_{N}, P_{N}\right)=\varphi\left(P_{N}\right)+\psi\left(P_{N}, \operatorname{sgn}\left(u_{N}\right)\right) u_{N}, V_{P}(y)=V_{0}+S y$, and $V_{N}(y)=V_{0}-S y$.

With $\varphi\left(P_{P}\right)$ and $\varphi\left(P_{N}\right)$ are a polynomial functions of the pressure, $\psi\left(P_{P}, \operatorname{sgn}\left(u_{P}\right)\right)$ and $\psi\left(P_{N}, \operatorname{sgn}\left(u_{N}\right)\right)$ are a polynomial functions of the pressure and the input control, $F_{e x t}(t)$ is the external force.

Where $y$ is the position, $v$ is the velocity, $u_{P}$ and $u_{N}$ are servo-distributors voltages, $P_{P}$ and $P_{N}$ are pressure in the chamber $P$ and $N, q_{m}$ mass flow rate provided from servo-distributor to cylinder chamber, $\varphi($.$) leakage poly-$ nomial function, $\psi(.,$.$) polynomial function, b_{v}$ viscous friction coefficient, $k$ polytropic constant, $M$ total load mass, $S$ piston section, $T$ temperature, $r$ perfect gas constant, $V_{0}$ half-cylinder volume.

Taken $\quad x=\left(x_{1}, x_{2}, x_{3}, x_{4}\right)^{T}=\left(y, v, P_{p}, P_{N}\right)^{T} \quad$ and $\left(u_{P}, u_{N}\right)^{T}=\left(u_{1}, u_{2}\right)^{T}$.

The state space model become

$\left\{\begin{array}{l}\dot{x}_{1}=x_{2} \\ \dot{x}_{2}=a x_{3}-a x_{4}-b_{v} x_{2}-f_{e x t}(t) \\ \dot{x}_{3}=f_{1}(x)+g_{1}(x) u_{1} \\ \dot{x}_{4}=f_{2}(x)+g_{2}(x) u_{2}\end{array}\right.$

with $f_{1}(x)=\frac{k r T}{V_{P}\left(x_{1}\right)}\left(\varphi\left(x_{3}\right)-\frac{S}{r T} x_{3} x_{2}\right), \quad f_{e x t}(t)=\frac{F_{e x t}(t)}{M}$,

$f_{2}(x)=\frac{k r T}{V_{N}\left(x_{1}\right)}\left(\varphi\left(x_{4}\right)+\frac{S}{r T} x_{4} x_{2}\right), \quad a=\frac{S}{M}$,

$g_{1}(x)=\frac{k r T}{V_{P}\left(x_{1}\right)} \psi\left(x_{3}, \operatorname{sgn}\left(u_{P}\right)\right)$,

$g_{2}(x)=\frac{k r T}{V_{N}\left(x_{1}\right)} \psi\left(x_{4}, \operatorname{sgn}\left(u_{N}\right)\right)$.

\section{Linear CDM control}

CDM control is one of algebraic approach with polynomial form, it allow getting required response in the time domain by arranging the poles of the closed loop transfer function using stability index and equivalent time constant.

The output of the closed-loop system is defined as

$y(s)=\frac{N(s) F(s)}{P(s)} \operatorname{ref}(s)+\frac{A(s) N(s)}{P(s)} d(s)$.

Where $y$ is the system output, ref is the reference input, $u$ is the control input and $d$ is the external disturbance signal, $N(s)$ and $D(s)$ are the numerator and the denominator of the transfer function of the plant, respectively, $A(s)$ and $B(s)$ are the denominator polynomial and the feedback numerator of the controller transfer function, while $F(s)$ is the pre-filter, $P(s)$ is the characteristic polynomial.

The stability index $\gamma_{i}$, the equivalent time constant $T_{0}$ and the stability limit $\gamma_{i}^{*}$ [16-18] are defined as $T_{0}=t_{s} /(2.5 \sim 3), \gamma_{i}=\mu_{i}^{2} /\left(\mu_{i-1} \mu_{i+1}\right)$ for $i=1$ to $(n-1)$, $\gamma_{i}^{*}=1 / \gamma_{i+1}+1 / \gamma_{i-1}, \quad \gamma_{1}=2.5, \quad \gamma_{i}=2$ for $i=2 \sim(n-1)$, $\gamma_{0}=\gamma_{n}=\infty$ with $t_{s}$ is the settling time. Finally the pre-filter 
$F(s)=P(0) / N(s)$ is used for reducing the steady state error and the characteristic polynomial is given in [16] by Eq. (4):

$$
P(s)=\mu_{0}\left[\left\{\sum_{i=2}^{n}\left(\prod_{j=1}^{i-1} 1 / \gamma_{i-j}^{j}\right)\left(T_{0} s\right)^{i}\right\}+T_{0} s+1\right] \text {. }
$$

\section{Command filtered CDM-backstepping and stability analysis}

This section deal with robust command filtered CDMbackstepping approach with an incorporated stability analysis for position tracking of electro-pneumatic system. The composition of this method is as follows: a CDMbackstepping procedure is used to derive the stabilizing functions and compensate the uncertainty generated by parameters variations and the external disturbances. As well, the command filter is used to guarantee that the desired state is closer to the value of the stabilizing function.

Initially we define the first tracking error as follows $e_{1}=x_{1}-x_{d}$ with $x_{d}$ is the reference, its time derivative along the trajectories of the system given by Eq. (2) can be written as $\dot{e}_{1}=x_{2}-\dot{x}_{d}$, by adding and subtracting the terms $\varphi_{1}$ and $\varphi_{1 f}$ from $x_{2}$, then its time derivative is given as $\dot{e}_{1}=\dot{x}_{1}-\dot{x}_{d}=x_{2}+\varphi_{1}-\varphi_{1}+\varphi_{1 f}-\varphi_{1 f}-\dot{x}_{d}$, otherwise $\dot{e}_{1}=\left(x_{2}-\varphi_{1 f}\right)+\varphi_{1}+\varphi_{1 f}-\varphi_{1}-\dot{x}_{d}$, as a consequence $\dot{e}_{1}=e_{2}+\varphi_{1}+\varphi_{1 f}-\varphi_{1}-\dot{x}_{d}$, with $\varphi_{1 f}$ represents the filtered command signal generated using the first order low pass filter with $\dot{\varphi}_{1 f}=\sigma_{1}\left(\varphi_{1}-\varphi_{1 f}\right)$, where $\sigma_{1}$ is the filter's cutoff frequency, whereas $\varphi_{1 f}(0)=\varphi_{1}\left(x_{10}, x_{d 0}, \dot{x}_{d 0}\right)$ denotes the initial value of the virtual control signal with $x_{10}=0$, $x_{d 0}=0$ and $\dot{x}_{d 0}=0$.

To compensate the influence of the command filter on the closed loop stability, a new variable $\varepsilon_{1}$ is introduced such that the compensated tracking error signal is defined as $z_{1}=e_{1}-\varepsilon_{1}$, then $\dot{z}_{1}=\dot{e}_{1}-\dot{\varepsilon}_{1}$, we then proceed by choosing the first Lyapunov function [8-10] as follows $V_{1}=0.5 z_{1}^{2}$, its time derivative is $\dot{V}_{1}=z_{1}\left(\dot{e}_{1}-\dot{\varepsilon}_{1}\right)$, to ensure the negativity of the time derivative of $V_{1}$, the virtual control [8] $\varphi_{1}$ and the dynamics of $\varepsilon_{1}$ are selected as $\varphi_{1}=-\lambda_{1} e_{1}+\dot{x}_{d}$, with $\dot{\varepsilon}_{1}=-\lambda_{1} \varepsilon_{1}+\varphi_{1 f}-\varphi_{1}+\varepsilon_{2}$ and $\varepsilon_{1}(0)=0$, where $\varepsilon_{2}$ is a new variable, then $\dot{z}_{1}=\dot{e}_{1}-\dot{\varepsilon}_{1}=e_{2}+\varphi_{1}+\varphi_{1 f}-\varphi_{1}-\dot{x}_{d}-\dot{\varepsilon}_{1}$, after that

$$
\begin{array}{r}
\dot{z}_{1}=\dot{e}_{1}-\dot{\varepsilon}_{1}=e_{2}+\varphi_{1}+\varphi_{1 f}-\varphi_{1}-\dot{x}_{d}+\lambda_{1} \dot{\varepsilon}_{1} \\
-\varphi_{1 f}+\varphi_{1}-\varepsilon_{2}=e_{2}-\varepsilon_{2}+\varphi_{1}-\dot{x}_{d}+\lambda_{1} \dot{\varepsilon}_{1} .
\end{array}
$$

Hence, $\dot{z}_{1}=z_{2}-\lambda_{1} z_{1}$, as a result $\dot{V}_{1}=-\lambda_{1} z_{1}^{2}+z_{1} z_{2}$.

In the second step of the controller design, considers the subsystem given by $\dot{x}_{2}=a x_{3}-a x_{4}-b_{v} x_{2}-f_{e x t}(t)$.
The second compensated tracking error signal is defined as $z_{2}=e_{2}-\varepsilon_{2}$, its derivative is $\dot{z}_{2}=\dot{e}_{2}-\dot{\varepsilon}_{2}$. The second error tracking is taking $e_{2}=x_{2}-\varphi_{1 f}$, we then proceed by compensating for the second command filter using the new vector $\varepsilon_{2}$ and define $z_{2}=e_{2}-\varepsilon_{2}$. The backstepping variable is $e_{2}=x_{2}-\varphi_{1 f}$, and its derivative is prearranged as $\quad \dot{e}_{2}=\dot{x}_{2}-\dot{\varphi}_{1 f}=a x_{3}-a x_{4}-b_{v} x_{2}-f_{e x t}(t)-\dot{\varphi}_{1 f}, \quad$ then $\dot{e}_{2}=a x_{3}-a x_{4}-b_{v} x_{2}+\varphi_{2}-\varphi_{2}+\varphi_{2 f}-\varphi_{2 f}-f_{e x t}(t)-\dot{\varphi}_{1 f}$, also we introduce the second Lyapunov function $V_{2}=0.5 z_{1}^{2}+0.5 z_{2}^{2}$, its derivative is given as $\dot{V}_{2}=z_{1} \dot{z}_{1}+z_{2} \dot{z}_{2}=-\lambda_{1} z_{1}^{2}+z_{1} z_{2}+z_{2}\left(\dot{e}_{2}-\dot{\varepsilon}_{2}\right)$.

As a result, it can be written by Eq. (6):

$$
\begin{aligned}
& \dot{V}_{2}=-\lambda_{1} z_{1}^{2}+z_{1} z_{2}+z_{2} \\
& \left(a x_{3}-a x_{4}-b_{v} x_{2}+\varphi_{2}-\varphi_{2}+\varphi_{2 f}-\varphi_{2 f}-f_{e x t}(t)-\dot{\varphi}_{1 f}-\dot{\varepsilon}_{2}\right) .
\end{aligned}
$$

Treat $a x_{3}-a x_{4}-b_{v} x_{2}$ as an independent control whose desired input is $\varphi_{2}=-z_{1}-\lambda_{2} e_{2}+b_{v} x_{2}+f_{\text {ext }}(t)+\dot{\varphi}_{1 f}$ with $\lambda_{2}>0$. Then, the virtual controls are expressed as $x_{3 d}=\varphi_{2 f} /(2 a)$ and $x_{4 d}=-\varphi_{2 f} /(2 a)$, where $\varphi_{2 f}$ symbolizes the filtered command signal produced by means of the first order low pass filter with $\dot{\varphi}_{2 f}=\sigma_{2}\left(\varphi_{2}-\varphi_{2 f}\right)$, $\varphi_{2 f}(0)=\varphi_{20}$, and $\sigma_{2}$ is the filter's cut-off frequency, whereas $\varphi_{2 f}(0)=\varphi_{1}\left(x_{10}, x_{20}, \varphi_{10}\right)$ denotes the initial value of the virtual control signal.

Therefore, the derivative of $V_{2}$ fulfils

$$
\begin{aligned}
\dot{V}_{2} & =-\lambda_{1} z_{1}^{2}+z_{1} z_{2} \\
& +z_{2}\left(a e_{3}-a e_{4}+\varphi_{2}+\varphi_{2 f}-\varphi_{2}-f_{e x t}(t)-\dot{\varphi}_{1 f}-\dot{\varepsilon}_{2}\right) .
\end{aligned}
$$

In order for $\dot{V}_{2}$ to be negative, the dynamics equation of $\varepsilon_{2}$ is selected as $\dot{\varepsilon}_{2}=-\lambda_{1} \varepsilon_{2}+\varphi_{2 f}-\varphi_{2}$ with $z_{3}=e_{3}$ and $z_{4}=e_{4}$, then $\dot{V}_{2}=-\lambda_{1} z_{1}^{2}-\lambda_{2} z_{2}^{2}+a z_{2} z_{3}-a z_{2} z_{4}$, at that time, we define an auxiliary variable $\zeta=\left(\begin{array}{ll}x_{3} & x_{4}\end{array}\right)^{T}$, one can obtain $\dot{\zeta}=F(x)+G(x) U$ with $U=\left(\begin{array}{ll}u_{1} & u_{2}\end{array}\right)^{T}$, $G(x)=\left(\begin{array}{lll}g_{1}(x) & g_{2}(x)\end{array}\right)^{T}$ and $F(x)=\left(\begin{array}{ll}f_{1}(x) & f_{2}(x)\end{array}\right)^{T}$.

Atfinal stepwehave $z_{3}=e_{3}=\zeta_{1}-x_{3 d}, z_{4}=e_{4}=\zeta_{2}-x_{4 d}$, then let the vector $E=\left(\begin{array}{ll}z_{3} & z_{4}\end{array}\right)^{T}$, afterwards

$E=\zeta-\zeta^{d}$ with $\zeta_{d}=\left(\begin{array}{ll}x_{3 d} & x_{4 d}\end{array}\right)^{T}$.

The control signal can be expressed by Eq. (9):

$A_{1}(x) U+A_{2}(x)(d U / d t)=E_{c}(t)$.

Where the error $E_{c}(t)$ can be specified by Eq. (10):

$E_{c}(t)=C_{0}(x) \zeta^{d}-B_{0}(x) \zeta-B_{1}(x) \dot{\zeta}$. 
$A_{1}(x), A_{2}(x), C_{0}(x), B_{0}(x)$ and $B_{1}(x)$ are nonlinear control gains and are designed as follows. Assuming that the constant gains $\delta_{1}, \delta_{2}, c_{1}$ and $c_{2}$ are such that

$\left|c_{i} \delta_{i} \operatorname{sgn}\left(z_{s i}\right) \int_{0}^{t} z_{i+2}(\theta) d \theta\right| \geq\left|a z_{2}\right|+\left|h_{i}(x)\right|$, with $i=1.2$.

We will develop the control signal $U=\left(\begin{array}{ll}u_{1} & u_{2}\end{array}\right)^{T}$ that force the errors $z_{3}$ and $z_{4}$ to converge to zero. Firstly let $\left\{\begin{array}{l}A_{1}(x)=-K d G(x) / d t \\ A_{2}(x)=-K G(x)\end{array}\right.$.

With $K$ is a diagonal matrix, by replacing Eq. (8) into Eq. (10), we obtain $E=\left(B_{0}^{-1} C_{0}-I\right) \zeta^{d}-B_{0}^{-1} E_{c}$, taking the gain matrix such that $C_{0}(x)=B_{0}(x)=C_{0}=\operatorname{diag}\left(c_{1}, c_{2}\right)$, $B_{1}(x)=\operatorname{diag}(0,0)$, then

$E_{c}=-C_{0} E$.

Its second derivative is given as

$\ddot{E}_{c}(t)=C_{0} \ddot{\zeta}^{d}(t)-C_{0} \ddot{\zeta}(t)$.

By combining Eq. (9), Eq. (10) and Eq. (12) yields

$\ddot{\zeta}(t)=\dot{F}(x)-K_{1} E_{c}$.

With $K_{1}=K^{-1}$, the insertion of Eq. (15) into Eq. (14) gives $\ddot{E}_{c}(t)=C_{0} \ddot{\zeta}^{d}(t)-C_{0}\left(\dot{F}(x)-K_{1} E_{c}\right)$, by integrating it, gives $\dot{E}_{c}(t)=C_{0} \dot{\zeta}^{d}(t)-C_{0}\left(F(x)-K_{1} \int_{0}^{t} E_{c}(\theta) d \theta\right)$, and using Eq. (13) provides

$\dot{E}(t)=H(x)+K_{1} \int_{0}^{t} E(\theta) d \theta$.

In addition, $\quad H(x)=F(x)-\dot{\zeta}_{d}(t)=\left(h_{1}(x), h_{2}(x)\right)^{T}$, $K_{2}=C_{0} K_{1}$, taking $K_{2}=\operatorname{diag}\left(\delta_{1} \operatorname{sgn}\left(z_{s 1}\right), \delta_{2} \operatorname{sgn}\left(z_{s 2}\right)\right)$, $z_{s 1}=z_{3} \int_{0}^{t} z_{3}(\theta) d \theta$ and $z_{s 2}=z_{4} \int_{0}^{t} z_{4}(\theta) d \theta$.

The final Lyapunov function is $V_{3}=V_{2}+0.5 z_{3}^{2}+0.5 z_{4}^{2}$, its derivative is specified by $\dot{V}_{3}=\dot{V}_{2}+z_{3} \dot{z}_{3}+z_{4} \dot{z}_{4}$, then using the expressions of $z_{3}(t)$ and $z_{4}(t)$, we achieve $\dot{V}_{3}=-\lambda_{1} z_{1}^{2}-\lambda_{2} z_{2}^{2}+a z_{2} z_{3}-a z_{2} z_{4}+z_{3} \dot{z}_{3}+z_{4} \dot{z}_{4}$, this provide $\dot{V}_{3}=-\lambda_{1} z_{1}^{2}-\lambda_{2} z_{2}^{2}+E^{T}\left(\dot{E}+\left(a z_{2},-a z_{2}\right)^{T}\right)$, replacing $\dot{E}$ by Eq. (16) we attain $\dot{V}_{3}=-\lambda_{1} z_{1}^{2}-\lambda_{2} z_{2}^{2}+\Lambda(t)$, where

$$
\Lambda(t)=E^{T}\left[\left(\begin{array}{c}
a z_{2} \\
-a z_{2}
\end{array}\right)+\left(\begin{array}{l}
h_{1}(x) \\
h_{2}(x)
\end{array}\right)-\left(\begin{array}{c}
c_{1} \delta_{1} z_{s 1} \operatorname{sgn}\left(z_{s 1}\right) \\
c_{2} \delta_{2} z_{s 2} \operatorname{sgn}\left(z_{s 2}\right)
\end{array}\right)\right] .
$$

If $\Lambda(t)<0$ then $\dot{V}_{3} \leq 0$, the gains $\delta_{1}, \delta_{2}, c_{1}$ and $c_{2}$ must be taken using inequality Eq. (11).

\section{ABC based command filtered CDM-backstepping}

The $\mathrm{ABC}$ is resulting from biological behavior of bee colony founded on the searching way of the honey bees [22], which is used to solve the optimal problem in the optimization process. Comparing with powerful and known meta-heuristics such as genetic algorithm GA and particle swarm optimization, PSO, the presented $\mathrm{ABC}$ algorithm habitually achieved better solutions in less time, because of this algorithm summarize the easiness, flexibility, employs less control parameters [23, 24], it can be efficiently used for solving multidimensional optimization problems [23, 25] and it can combines a global search with a local search [23], while GA uses only global search. The ABC optimization technique has three categories of bees: employed bee, onlooker bee, and scout bee [19-21]. The first half of the colony is the population size is constituted of one half of employed bees and another half of onlooker bees. The number of employed bees is the same to that of food source which is obtainable in the natural world. In particular, each food source is used by one employed bee. Onlooker obtains information concerning food sources via dances of employed bees [25]. A selection is done by onlookers for selecting an employed bee and to exploit the food source to gather nectar [25]. The employed bee becomes scout if it was abandoned from food source which supports in exploring more potential food sources [26-28]. In ABC algorithm, the position of a food source symbolizes a probable solution to be optimized. However the fitness of the optimized solution related to the source is termed as nectar amount.

The key steps of $\mathrm{ABC}$ algorithm are as follows:

STEP 1: The population is produced randomly between the search spaces, is given in $[24,26]$ by Eq. (18):

$$
\operatorname{Par}_{i}^{j}=\operatorname{Par}_{j}^{\min }+\operatorname{rand}(0,1) \times\left(\operatorname{Par}_{j}^{\max }-\operatorname{Par}_{j}^{\min }\right) \text {. }
$$

Where $i=1, \ldots, N$ denote the number of food sources (equal to half to entire number of bees) and $j=1, \ldots, D$. $N$ and $D$ signify the dimension (number of controller parameters) of the problem, $P a r^{\max }$ and $P a r^{\min }$ are the upper bound and the lower bound of the search space.

Step 2: In this step, in the surrounding area of the solution, each employee bee searches a new candidate solution $[24,26]$ via $v_{i}^{j}=\operatorname{Par}_{j}^{i}+\operatorname{rand}(-1,1) \times\left(\operatorname{Par}_{j}^{i}-\operatorname{Par}_{j}^{k}\right)$, where the constant $k$ is chosen randomly neighbor of $i$, where $k \neq i$ and $j$ is a dimension designated randomly. Once the solution $v_{i}$ is discovered, it is compared to $\operatorname{Par}_{i}$. If $v_{i}$ is better than $\operatorname{Par}_{i}$, Par $_{i}$ is substituted by $v_{i}$ and trial 
counter of $i$ is returned to zero. In addition, the trial counter of $i$ is simply incremented by one, if Par is better than $v_{i}$.

Step 3: In the hive, when the dance is performed by the employed bees, an onlooker bee chooses her food source to utilize, probabilistically, corresponding to the solution the probability [28] of food sources is considered by Eq. (19):

$$
P_{i}=f_{i t} / \sum_{j=1}^{m} f i t_{j} .
$$

The fitness function is designated by fit $_{i}=1 /(1+J)$, where $J$ is the objective function to be optimized and given by Eq. (20):

$$
J=\sum_{i=1}^{4} \int_{0}^{\infty} z_{i}^{2} d t .
$$

After choosing probabilistically the food source, the onlooker bee behaves in similar fashion as declared in the employed step.

Step 4: After employed and onlooker steps, all solutions of the trial counters are examined, and if they exceed the maximum limit, the employed bee of that solution becomes scout and that solution is abandoned. Subsequently, those scouts generate a new solution by Eq. (18) to substitute the abandoned source.

\section{Computer simulations}

In order to study the efficiency of the ABC optimization in the position control, we have designed a computer simulation of optimized controller where compared with the traditional controller, the test was divided in two phases ideal case and robustness analysis in the case when the disturbance and uncertainties are added to the system. The parameters of the system are $b=50 \mathrm{~N} / \mathrm{m} / \mathrm{s}, V_{D N}=1.2 \times 10^{-5} \mathrm{~m}^{2}$, $T=293 \mathrm{~K}, \quad V_{0}=3.4 \times 10^{-4} \mathrm{~m}^{3}, \quad r=287.1 \mathrm{~J}_{\mathrm{kg}}{ }^{-1} \cdot \mathrm{K}^{-1}$, $S=0.0045 \mathrm{~m}^{2}, \quad M=3.4 \mathrm{~kg}, k=1.2$. The initial values are $y(0)=-0.07 \mathrm{~m}, v(0)=0 \mathrm{~ms}^{-1}, P_{P}(0)=1 \mathrm{bar}$. The search space of controller parameters are $0<\lambda_{1}<100$, $0<\lambda_{2}<100, \quad 0<\lambda_{3}<100, \quad 0<\delta_{1}<50, \quad 0<\delta_{2}<50$, $0<c_{1}<50,0<c_{2}<50,0<\sigma_{1}<30,0<\sigma_{2}<30$.

\subsection{First test scenario}

The results are represented in Figs. 1-3, which display comparisons between the performance of the conventional controller and the optimized controller. From Fig. 1, it is clear that the system with conventional controller ensure high settling time, high steady state error and ISE value with $4.12 \mathrm{~mm}$, and $1.18 \mathrm{~mm}$ respectively. The optimized controller enhances the performance where the settling time and

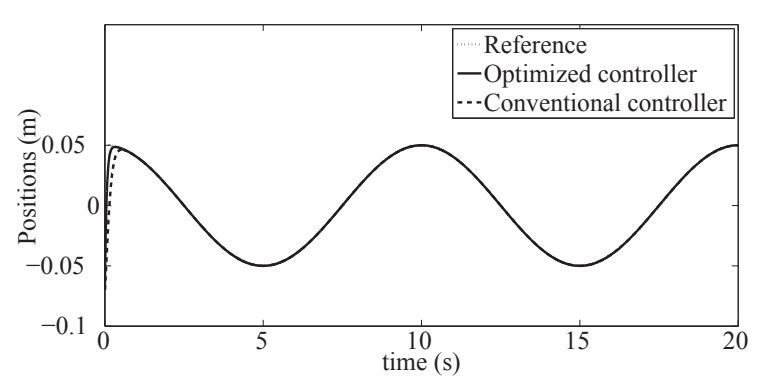

Fig. 1 First test, actual positions for both controllers

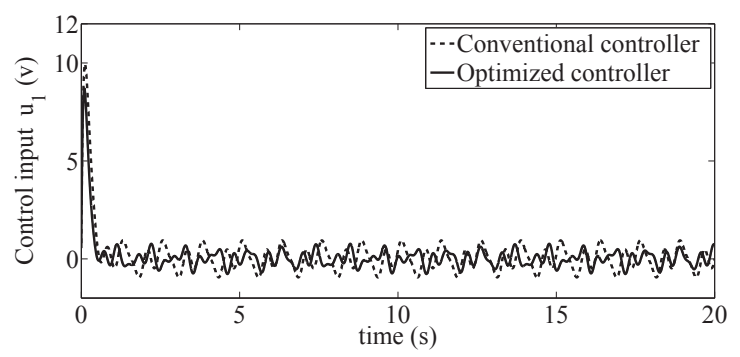

Fig. 2 First test, actual controls inputs for both controllers

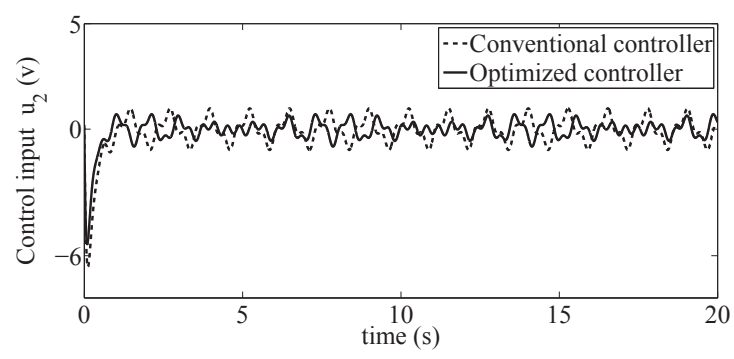

Fig. 3 First test, actual controls inputs for both controllers

steady state error are extremely minimized with $0.097 \mathrm{~mm}$ and $0.021 \mathrm{~mm}$ respectively. The overshoot of the system is nearly equal zero for both controllers; the results obtained are depicted in Table 1. Fig. 2 and Fig. 3 show the comparison of the actuators controls action where the amplitude of control input is less, then very suitable for the optimized controller. So, tuning of filtered CDM-backstepping gains is useful in minimizing the settling time, steady state error and control effort but has no effect in overshoot.

Table 1 Performance comparison of algorithms

\begin{tabular}{lcc}
\hline & Conventional controller & Optimized controller \\
\hline & $\lambda_{1}=80, \lambda_{2}=40$, & $\lambda_{1}=60.2, \lambda_{2}=35.1$, \\
$\lambda_{3}=27$, & $\lambda_{3}=21.4$, \\
Controller & $\delta_{1}=18, \delta_{2}=17$, & $\delta_{1}=15.2, \delta_{2}=8.32$, \\
parameters & $c_{1}=10, c_{2}=11$, & $c_{1}=9.43, c_{2}=10.1$, \\
& $\sigma_{1}=20, \sigma_{2}=20$ & $\sigma_{1}=24.3, \sigma_{2}=25.2$ \\
Settling time & $t_{s}=0.71 \mathrm{~s}$ & $t_{s}=0.41 \mathrm{~s}$ \\
$\begin{array}{l}\text { Performance } \\
\text { criteria }\end{array}$ & $J=0.00412$ & $J=0.00097$ \\
\hline
\end{tabular}




\subsection{Second test scenario}

To show the superiority and the robustness of the proposed $\mathrm{ABC}$ technique for optimizing the controller parameters, wide changes are effected in the operating conditions and system parameters by adding the external disturbance and varying system parameters from the nominal values in the range of $-25 \%$ to $25 \%$ applied at $t=0 \mathrm{~s}$. Figs. $4-6$ illustrate the performance comparison of the controllers, the results obtained from the optimized and conventional controllers are compared, minimum steady state error, settling time, ISE values and control efforts are obtained with optimized controller and the effect on the system performance is negligible. Hence, the proposed control is the best in robustness under these changes.

\section{Conclusion}

The proposed paper applies a new optimization method for optimal gain tuning of controller parameters by means of $\mathrm{ABC}$ algorithm in order to obtain high performance of the electro-pneumatic system using command filtered CDMbakstepping to avoid the problem of increase of complexity, the asymptotic stability is proven and guaranteed using Lyapunov theory, the optimization method is designed with the comprehensive analysis of its tuning performance and its impact to robustness, throughout the searching evolution, the well-defined objective function is successively minimized so that the optimal control parameters finally ensued, in the simulation, two types of control cases are provided to display the feasibility of the proposed method, where the closed-loop control performances of the optimized controller are compared with traditional controller, the asymptotic convergence is achieved for both control system without overshoot, where the optimized control system has best performance and is not affected by inserted disturbance and changes of the parameters values, when the considered system parameters are varied in the

\section{References}

[1] Lin, Z., Zhang, T., Xie, Q., Wei, Q. "Intelligent Electro-Pneumatic Position Tracking System Using Improved Mode-Switching Sliding Control With Fuzzy Nonlinear Gain", IEEE Access, 6, pp. 34462-34476, 2018.

https://doi.org/10.1109/ACCESS.2018.2847637

[2] Ayadi, A., Hajji, S., Smaoui, M., Chaari, A., Farza, M. "Experimental sensorless control for electropneumatic system based on high gain observer and adaptive sliding mode control", The International Journal of Advanced Manufacturing Technology, 93(9-12), pp. 4075-4088, 2017. https://doi.org/10.1007/s00170-017-0885-x

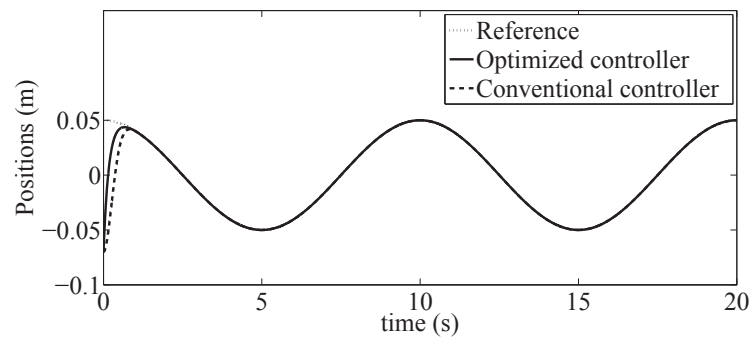

Fig. 4 Second test, actual positions for both controllers

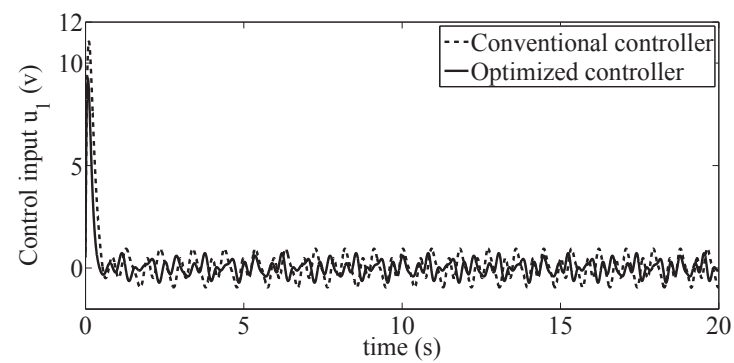

Fig. 5 Second test, actual controls inputs for both controllers

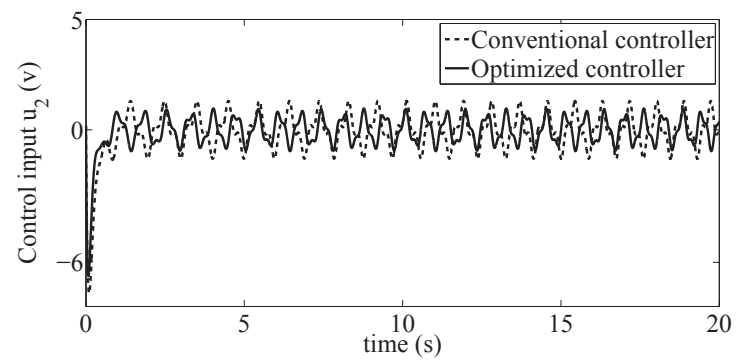

Fig. 6 Second test, actual controls inputs for both controllers

range of $\pm 25 \%$. Consequently it is seen from this study, that this optimization technique can be applied to the electro-pneumatic system successfully. Then simulation results expose the superiority of the optimized controller design method. Also, for future research, it is worthwhile to valid experimentally the research method.

[3] Yan, X., Primot, M., Plestan, F. "Electropneumatic actuator position control using second order sliding mode", Elektrotechnik \& Informationstechnik, 133(6), pp. 266-273, 2016. https://doi.org/10.1007/s00502-016-0423-9

[4] Dólleman, P., Carneiro, J. F., Gomes de Almeida, F. "Exploring the use of two servo-valves for servo-pneumatic control", The International Journal of Advanced Manufacturing Technology, 97(9-12), pp. 3963-3980, 2018. https://doi.org/10.1007/s00170-018-2230-4 
[5] Al-Saloum, S., Taha, A., Chouaib, I. "Comparative Study of Electro-pneumatic Actuator Performance Using Linear, Sliding Mode and Hybrid Position Control Laws", WSEAS Transactions on Systems, 17, pp. 1-15, 2018. [online] Available at: http://www. wseas.org/multimedia/journals/systems/2018/a025902-770.pdf [Accessed: 28 February 2017]

[6] Mechter, A., Kemih, K., Ghanes, M. "Backstepping control of a wind turbine for low wind speeds", Nonlinear Dynamic, 84(4), pp. 2435-2445, 2016. https://doi.org/10.1007/s11071-016-2655-y

[7] Li, X., Chen, X., Zhou, C. "Combined Observer-Controller Synthesis for Electro-Hydraulic Servo System with Modeling Uncertainties and Partial State Feedback", Journal of the Franklin Institute, 355(13), pp. 5893-5911, 2018.

https://doi.org/10.1016/j.jfranklin.2018.05.050

[8] Arsalan, M., Iftikhar, R., Ahmad, I., Hasan, A., Sabahat, K., Javeria, A. "MPPT for photovoltaic system using nonlinear backstepping controller with integral action", Solar Energy, 170, pp. 192-200, 2018 . https://doi.org/10.1016/j.solener.2018.04.061

[9] Liu, Y., Liu, X., Jing, Y., Zhou, S. "Adaptive backstepping $\mathrm{H}_{\infty}$ tracking control with prescribed performance for internet congestion", ISA Transactions, 72, pp. 92-99, 2018. https://doi.org/10.1016/j.isatra.2017.10.004

[10] Dashkovskiy, S. N., Pavlichkov, S. S. "Integrator backstepping for uncertain nonlinear systems with non-smooth dynamics", European Journal of Control, 40, pp. 68-79, 2018. https://doi.org/10.1016/j.ejcon.2017.12.002

[11] Li, C., Zhang, Y., Li, P. "Full control of a quadrotor using parameter-scheduled backstepping method: implementation and experimental tests", Nonlinear Dynamic, 89(2), pp. 1259-1278, 2017. https://doi.org/10.1007/s11071-017-3514-1

[12] Yu, J., Shi, P., Zhao, L. "Finite-time command filtered backstepping control for a class of nonlinear systems", Automatica, 92, pp. $173-180,2018$. https://doi.org/10.1016/j.automatica.2018.03.033

[13] Aboudonia, A., El-Badawy, A., Rashad, R. "Active anti-disturbance control of a quadrotor unmanned aerial vehicle using the command-filtering backstepping approach", Nonlinear Dynamics, 90(1), pp. 581-597, 2017. https://doi.org/10.1007/s11071-017-3683-y

[14] Wu, G., Meng, X. "Nonlinear disturbance observer based robust backstepping control for a flexible air-breathing hypersonic vehicle", Aerospace Science and Technology, 54, pp. 174-182, 2016. https://doi.org/10.1016/j.ast.2016.04.018

[15] Mohamed, T. H., Shabib, G., Ali, H. "Distributed load frequency control in an interconnected power system using ecological technique and coefficient diagram method", International Journal of Electrical Power and Energy Systems, 82, pp. 496-507, 2016. https://doi.org/10.1016/j.ijepes.2016.04.023

[16] Mohamed, T. H., Diab, A. A. Z., Hussein, M. M. "Application of Linear Quadratic Gaussian and Coefficient Diagram Techniques to Distributed Load Frequency Control of Power Systems", Applied Sciences, 5(4), pp. 1603-1615, 2015. https://doi.org/10.3390/app5041603
[17] Ali, R., Mohamed, T. H., Qudaih, Y. S., Mitani, Y. "A new load frequency control approach in an isolated small power systems using coefficient diagram method", International Journal of Electrical Power and Energy Systems, 56, pp. 110-116, 2014. https://doi.org/10.1016/j.ijepes.2013.11.002

[18] Erkan, K., Yalçın, B. C., Garip, M. "Three-axis gap clearance I-PD controller design based on coefficient diagram method for 4-pole hybrid electromagnet", Automatika, 58(2), pp. 147-167, 2017. https://doi.org/10.1080/00051144.2017.1382649

[19] Chang, W.-D. "Nonlinear CSTR control system design using an artificial bee colony algorithm", Simulation Modelling Practice and Theory", 31, pp. 1-9, 2013. https://doi.org/10.1016/j.simpat.2012.11.002

[20] Rajasekhar, A., Jatoth, R. K., Abraham, A. "Design of intelligent $\mathrm{PID} / \mathrm{PI}^{\lambda} \mathrm{D}^{\mu}$ speed controller for chopper fed DC motor drive using opposition based artificial bee colony algorithm", Engineering Applications of Artificial Intelligence, 29, pp. 13-32, 2014. https://doi.org/10.1016/j.engappai.2013.12.009

[21] Kumar, A., Kumar, D., Jarial, S. K. "A Review on Artificial Bee Colony Algorithms and Their Applications to Data Clustering", Cybernetics and Information Technologies, 17(3), pp. 3-28, 2017. https://doi.org/10.1515/cait-2017-0027

[22] Sreejith, S., Simon, S. P. "Security Constrained Unit Commitment Incorporating Interline Power Flow Controller", Periodica Polytechnica Electrical Engineering and Computer Science, 61(1), pp. 22-33, 2017. https://doi.org/10.3311/PPee.9460

[23] Karaboga, D., Akay, B. "A comparative study of Artificial Bee Colony algorithm", Applied Mathematics and Computation, 214(1), pp. 108-132, 2009. https://doi.org/10.1016/j.amc.2009.03.090

[24] Karaboga, D., Ozturk, C., Karaboga, N., Gorkemli, B. "Artificial bee colony programming for symbolic regression", Information Sciences, 209, pp. 1-15, 2012. https://doi.org/10.1016/j.ins.2012.05.002

[25] Karaboga, D., Basturk, B. "A powerful and efficient algorithm for numerical function optimization: artificial bee colony $(\mathrm{ABC})$ algorithm", Journal of Global Optimization, 39(3), pp. 459-471, 2007. https://doi.org/10.1007/s10898-007-9149-x

[26] Karaboga, D., Akay, B. "Proportional-Integral-Derivative Controller Design by Using Artificial Bee Colony, Harmony Search, and the Bees Algorithms", Proceedings of the Institution of Mechanical Engineers, Part I: Journal of Systems and Control Engineering, 224(7), pp. 869-883, 2010. https://doi.org/10.1243/09596518JSCE954

[27] Karaboga, D., Gorkemli, B., Ozturk, C., Karaboga, N. "A comprehensive survey: artificial bee colony $(\mathrm{ABC})$ algorithm and applications", Artificial Intelligence Review, 42(1), pp. 21-57, 2014. https://doi.org/10.1007/s10462-012-9328-0

[28] Karaboga, D., Ozturk, C. "A novel clustering approach: Artificial Bee Colony (ABC) algorithm", Applied Soft Computing, 11(1), pp. 652-657, 2011. https://doi.org/10.1016/j.asoc.2009.12.025 